\title{
Mental health outcomes among front and second line health workers associated with the COVID-19 pandemic in Italy.
}

\author{
Rodolfo Rossi, $M D^{1}$, Valentina Socci, PhD ${ }^{2}$, Francesca Pacitti, $M D^{2}$, Giorgio Di Lorenzo, MD ${ }^{1}$, Antinisca Di \\ Marco, $P h D^{3}$, Alberto Siracusano, $M D^{l}$ and Alessandro Rossi, $M D^{2}$. \\ 1 Chair of Psychiatry, Department of Systems Medicine, University of Rome Tor Vergata, Rome, Italy \\ 2 Chair of Psychiatry, Department of Applied Clinical Sciences and Biotechnologies, University of L'Aquila, L'Aquila, Italy \\ 3 Department of information engineering, computer science and mathematics, University of L'Aquila, L'Aquila, Italy
}

Correspondence:

Rodolfo Rossi

rudy86.rossi@gmail.com

\section{Introduction}

It has been recently shown that health workers (HWs) involved with the COVID-19 pandemic in Whuan and the Hubei province are exposed to high levels of stressful or traumatic events and express substantial negative mental health outcomes ${ }^{1}$, including stress-related symptoms, depression, anxiety and insomnia.

To the best of our knowledge, no data on HWs involved with the COVID-19 pandemic in Europe are available so far.

In this study, we report on mental health outcomes, using an on-line survey, among HWs in Italy.

\section{Methods}

Cross-sectional web-based observational study. Data on mental health were collected between March $27^{\text {th }}$ and March $31^{\text {th }} 2020$ using an on-line questionnaire spread throughout social networks, using a snowball technique along with sponsored social network advertisement. According to Italian public health organizations, the sampling period corresponded to the days immediately preceding the COVID-19 contagion peak, associated with a high level of healthcare system overwhelming. All HWs reporting to work in Italy were eligible. The questionnaire investigated key demographic variables, workplace characteristics, such as being a front-line or second-line worker, and information regarding the direct impact of the COVID-19, including having colleagues infected or deceased. Key mental health outcomes were Post-Traumatic Stress Disorder symptoms (PTSD), severe depression, anxiety, 
insomnia and perceived stress. These were assessed using the Italian version of the Global Psychotrauma Screen (GPS) ${ }^{2}$, the 9-item Patient Health Questionnaire (PHQ-9) ${ }^{3}$, the 7-item

Generalized Anxiety Disorder scale (GAD-7) ${ }^{4}$, the 7-item Insomnia Severity Index (ISI) ${ }^{5}$, and the 10item Perceived Stress Scale (PSS) ${ }^{6}$. Participants were classified as endorsing the aforementioned condition according to the following cut-offs: suspect PTSD $\geq 3$ on the GPS-PTSD subscale, severe depression if $\mathrm{PHQ} \geq 15$, severe anxiety if $\mathrm{GAD} \geq 15$, severe insomnia if $\mathrm{ISI} \geq 22$. Because the PSS does not represent any official disorder, a quartile split was used to separate the first quartile from the remaining participants.

A seemingly unrelated multivariable logistic regression model was fitted in order to explore the impact of gender, age, first-line working position, occupation, self and colleagues' exposure to contagion on the selected outcomes. Seemingly unrelated regression models allow to jointly model correlated outcomes.

Approval for this study was obtained from the local IRB at University of L'Aquila. On-line consent was obtained from the participants.

\section{Results}

A total of 1379 HWs completed the questionnaire. Because of the web-based snowball sampling strategy, response rate could not be calculated. Sample characteristics are reported in table 1. PTSD symptoms, severe depression, anxiety and insomnia, and high perceived stress were endorsed respectively by 681 (49.38\%), 341 (24.73\%), $273(19.80 \%), 114(8.27 \%)$ and $302(21.90 \%)$ respondents (Table 1$)$.

18 participants were excluded from regression analysis due to missing data. Regression analysis show that younger age and female gender were associated with all of the investigated outcomes, except for insomnia. Being a front-line HWs was specifically associated with PTSD symptoms. General Practitioners were more likely to endorse PTSD symptoms, nurses and health care assistants were more likely to endorse severe insomnia. Having a colleague deceased, hospitalized or in quarantine was associated with PTSD symptoms, depression, insomnia and perceived stress. Being exposed to contagion was associated with depression (Table 2). 
medRxiv preprint doi: https://doi.org/10.1101/2020.04.16.20067801; this version posted April 22, 2020. The copyright holder for this preprint

(which was not certified by peer review) is the author/funder, who has granted medRxiv a license to display the preprint in perpetuity.

It is made available under a CC-BY-NC-ND 4.0 International license .

\section{Discussion}

This is the first report on mental health outcomes and associated risk factors among HWs associated

with the COVID-19 pandemic in Italy. These results are in line with prior reports from China,

confirming a substantial proportion of health workers involved with the COVID-19 pandemic having

mental health issues, in particular young women, first-line HWs. Our results warrant further

monitoring and specific interventions on health workers throughout the COVID-19 pandemic in order

to prevent long-term mental-health related disabilities.

\section{Acknowledgments}

This work is supported by Territori Aperti, a project founded by "Fondo Territori Lavoro e

Conoscenza CGIL CISL UIL”.

Bibliography

1. Lai J, Ma S, Wang Y, et al. Factors Associated With Mental Health Outcomes Among Health Care Workers Exposed to Coronavirus Disease 2019. JAMA Netw open. 2020;3(3):e203976.

doi:10.1001/jamanetworkopen.2020.3976

2. Olff M, Aakvaag HF, Brewer D, et al. Screening for consequences of trauma - an update on the Global Collaboration on Traumatic Stress. Eur J Psychotraumatol. 11.

3. Spitzer RL, Kroken K, Williams JB. Validation and Utility of a Self-Report Version of PRIME-MD: The PHQ Primary Care Study. Primary Care Evaluation of Mental Disorders. Patient Health Questionnaire. JAMA. 1999;282(18):1737. doi:10.1001/jama.282.18.1737

4. Spitzer RL, Kroenke K, Williams JBW, Löwe B. A Brief Measure for Assessing Generalized Anxiety Disorder. Arch Intern Med. 2006;166(10):1092. doi:10.1001/archinte.166.10.1092

5. Morin CM, Belleville G, Bélanger L, Ivers H. The Insomnia Severity Index: Psychometric Indicators to Detect Insomnia Cases and Evaluate Treatment Response. Sleep. 2011;34(5):601-608. doi:10.1093/sleep/34.5.601

6. Cohen S, Hoberman HM. Positive Events and Social Supports as Buffers of Life Change Stress1. J Appl Soc Psychol. 1983;13(2):99-125. doi:10.1111/j.1559-1816.1983.tb02325.x 
medRxiv preprint doi: https://doi.org/10.1101/2020.04.16.20067801; this version posted April 22, 2020. The copyright holder for this preprint (which was not certified by peer review) is the author/funder, who has granted medRxiv a license to display the preprint in perpetuity.

It is made available under a CC-BY-NC-ND 4.0 International license .

Table 1: Sample characteristics

Overall

North

Center

South

\begin{tabular}{|c|c|c|c|c|}
\hline & No. $(\%) /$ Median $(I Q R)$ & No. $(\%) /$ Median $(I Q R)$ & No. $(\%) /$ Median $(I Q R)$ & No. $(\%) /$ Median $(I Q R)$ \\
\hline No. & 1379 & $667 / 1359(48.3 \%)$ & $412 / 1359(29.8 \%)$ & $280 / 1359(20.3 \%)$ \\
\hline \multicolumn{5}{|l|}{ Gender } \\
\hline Women & $1064 / 1379(77.2 \%)$ & $533 / 667(79.9 \%)$ & $321 / 412(77.9 \%)$ & $194 / 280(69.3 \%)$ \\
\hline Men & $315 / 1379(22.8 \%)$ & $134 / 667(20.1)$ & $91 / 412(22.1 \%)$ & $86 / 280(30.7 \%)$ \\
\hline Age & $39.0(16.0)$ & $38.0(16.0)$ & $(41.0)(16.0)$ & $38.0(15.0)$ \\
\hline \multicolumn{5}{|l|}{ Working Position } \\
\hline Front-line & $725(52.57 \%)$ & $448 / 667(67.17 \%)$ & $181 / 412(43.93 \%)$ & $88 / 279(31.43 \%)$ \\
\hline Second-line & $653(47.35 \%)$ & $219 / 667(32.83 \%)$ & $231 / 412(56.07 \%)$ & $191 / 279(68.21 \%)$ \\
\hline \multicolumn{5}{|l|}{ Occupation } \\
\hline Nurse & $472 / 1378(34.23 \%)$ & $265 / 667(39.7 \%)$ & $131 / 412(31.8 \%)$ & $67 / 279(23.93 \%)$ \\
\hline Physician & $433 / 1378(31.40 \%)$ & $163 / 667(24.44 \%)$ & $164 / 412(39.81 \%)$ & $100 / 279(35.71 \%)$ \\
\hline$G P$ & $86 / 1378(6.24 \%)$ & $35 / 667(5.25 \%)$ & $22 / 412(5.34 \%)$ & $28 / 279(10.00 \%)$ \\
\hline Other & 275/1378 (19.94\%) & $137 / 667(20.54 \%)$ & $77 / 412(18.69 \%)$ & $58 / 279(20.71 \%)$ \\
\hline$H C A$ & $112 / 1378(8.12 \%)$ & $67 / 667(10.04 \%)$ & $18 / 412(4.37 \%)$ & $26 / 279(9.29 \%)$ \\
\hline \multicolumn{5}{|l|}{ Education Level } \\
\hline Undergraduate & $222 / 1373(16.10 \%)$ & 131/667 (19.64\%) & $49 / 410(11.95 \%)$ & $36 / 276(13.04 \%)$ \\
\hline Postgraduate & $1151 / 1373(83.47 \%)$ & $536 / 667(80.36 \%)$ & $361 / 410(88.05 \%)$ & $240 / 276(86.96 \%)$ \\
\hline GPS-PTSD $\geq 3$ & $681 / 1376(49.38 \%)$ & $352(52.77 \%)$ & $193(46.84 \%)$ & $127 / 280(45.85 \%)$ \\
\hline PHQ $\geq 15$ & $341 / 1378(24.73 \%)$ & $182 / 666(27.29 \%)$ & $99 / 412(24.03 \%)$ & $55 / 280(19.64 \%)$ \\
\hline GAD $\geq 15$ & $273 / 1378(19.80 \%)$ & $130 / 666(19.49 \%)$ & $84 / 412(20.39 \%)$ & $55 / 280(19.64 \%)$ \\
\hline ISI $\geq \mathbf{2 2}$ & $114 / 1378(8.27 \%)$ & $66 / 667(9.90 \%)$ & $28 / 412(6.80 \%)$ & $17 / 280(6.07 \%)$ \\
\hline PSS $1^{\text {st }}$ quartile & $302 / 1378(21.90 \%)$ & $160 / 667(23.99 \%)$ & $86 / 412(20.87 \%)$ & $52 / 280(18.57 \%)$ \\
\hline GPS total score & $9(6)$ & $9(6)$ & $9(6)$ & $8(7)$ \\
\hline PHQ total score & $10(9)$ & $10(9)$ & $10(9)$ & $8(8)$ \\
\hline GAD total score & $9(9)$ & $9(8)$ & $9(10)$ & $8(9.5)$ \\
\hline ISI total score & $10(12)$ & $11(12)$ & $10(10.5)$ & $8(13)$ \\
\hline PSS total score & $24(11)$ & $24(10)$ & $24(9)$ & $22(12)$ \\
\hline
\end{tabular}

North: Aosta Valley, Piedmont, Liguria, Lombardy, Trentino-Alto Adige, Veneto, Friuli-Venezia Giulia, Emilia Romagna; Center: Tuscany, Marche, Umbria, Lazio; South: Abruzzo, Molise, Campania, Apulia, Basilicata, Calabria, Sicily, Sardinia. GP: General Practitioner; HCA: Health Care Assistant; GPS-PTSD: Global Psychotrauma Scale - post traumatic stress disorder subscale; PHQ: Patient Health Questionnaire; GAD: Generalized anxiety disorder scale; ISI: Insomnia Severity Index; PSS: Perceived Stress Scale; IQR: Interquartile range. 
Table 2: Seemingly Unrelated Logistic Regression Analysis

\begin{tabular}{|c|c|c|c|c|c|c|c|c|c|c|}
\hline \multirow[t]{2}{*}{$N=1369$} & \multicolumn{2}{|c|}{ GPS - PTSD } & \multicolumn{2}{|c|}{ PHQ } & \multicolumn{2}{|c|}{ GAD } & \multicolumn{2}{|r|}{ ISI } & \multicolumn{2}{|c|}{ PSS } \\
\hline & $O R$ & $95 \% C I$ & $O R$ & $95 \% C I$ & $O R$ & $95 \% C I$ & $O R$ & $95 \% C I$ & $O R$ & $95 \% C I$ \\
\hline Age std & $0.69 * *$ & {$[0.53,0.88]$} & $0.74 *$ & {$[0.56,0.98]$} & $0.60 * *$ & {$[0.44,0.82]$} & 0.71 & {$[0.46,1.09]$} & $0.63 * *$ & {$[0.46,0.85]$} \\
\hline \multicolumn{11}{|l|}{ Gender } \\
\hline Male & 1.00 (ref) & {$[1.00,1.00]$} & - & & - & & - & & - & \\
\hline Female & $2.31 * * *$ & {$[1.76,3.05]$} & $2.03 * * *$ & {$[1.44,2.87]$} & $2.18 * * *$ & {$[1.49,3.19]$} & 1.38 & {$[0.82,2.33]$} & $2.64 * * *$ & {$[1.80,3.87]$} \\
\hline \multicolumn{11}{|l|}{ Working Position } \\
\hline Second-line & 1.00 (ref) & {$[1.00,1.00]$} & - & & - & & - & & - & \\
\hline First-line & $1.37 *$ & {$[1.05,1.80]$} & 1.04 & {$[0.76,1.42]$} & 1.13 & {$[0.80,1.59]$} & 1.27 & {$[0.78,2.07]$} & 1.16 & {$[0.84,1.60]$} \\
\hline \multicolumn{11}{|l|}{ Occupation } \\
\hline Other $H W$ & 1.00 (ref) & {$[1.00,1.00]$} & - & & - & & - & & - & \\
\hline Nurse & 1.12 & {$[0.81,1.55]$} & 1.36 & {$[0.95,1.96]$} & 1.09 & {$[0.74,1.61]$} & $2.03 *$ & {$[1.14,3.59]$} & 0.74 & {$[0.51,1.08]$} \\
\hline Physician & 1.20 & {$[0.86,1.67]$} & 0.71 & {$[0.48,1.05]$} & 0.96 & {$[0.64,1.44]$} & 0.89 & {$[0.46,1.72]$} & 0.75 & {$[0.51,1.11]$} \\
\hline$G P$ & $1.75^{*}$ & {$[1.03,2.97]$} & 0.98 & {$[0.53,1.82]$} & 1.05 & {$[0.53,2.08]$} & 1.47 & {$[0.56,3.87]$} & 1.18 & {$[0.66,2.11]$} \\
\hline$H C A$ & 0.95 & {$[0.60,1.52]$} & 1.18 & {$[0.70,1.98]$} & 1.05 & {$[0.60,1.84]$} & $2.34 *$ & {$[1.06,5.18]$} & 0.59 & {$[0.33,1.05]$} \\
\hline \multicolumn{11}{|l|}{ Colleagues affected } \\
\hline No affected colleagues & 1.00 (ref) & {$[1.00,1.00]$} & - & & - & & - & & - & \\
\hline Deceased colleague & $2.60 * *$ & {$[1.30,5.19]$} & $2.07 *$ & {$[1.05,4.07]$} & 0.97 & {$[0.41,2.29]$} & $2.94 *$ & {$[1.21,7.18]$} & 1.84 & {$[0.88,3.87]$} \\
\hline Infected and hospitalized & $1.54 *$ & {$[1.10,2.16]$} & 1.39 & {$[0.95,2.03]$} & 1.18 & {$[0.78,1.77]$} & 1.14 & {$[0.66,1.96]$} & $1.93 * *$ & {$[1.30,2.85]$} \\
\hline Infected and in quarantine & $1.59 * * *$ & {$[1.21,2.09]$} & 1.38 & {$[1.00,1.90]$} & 1.19 & {$[0.85,1.67]$} & 0.88 & {$[0.54,1.45]$} & $1.66^{* *}$ & {$[1.19,2.32]$} \\
\hline Not exposed to contagion & 1.00 (ref) & {$[1.00,1.00]$} & & & & & & & & \\
\hline Exposed to contagion & 1.23 & {$[0.93,1.62]$} & $1.54 *$ & {$[1.11,2.14]$} & 1.14 & {$[0.81,1.62]$} & 1.45 & {$[0.88,2.39]$} & 1.01 & {$[0.73,1.41]$} \\
\hline
\end{tabular}

